\title{
MECHANICAL PROPERTIES OF HIP CAPSULE TISSUE AFTER A HIP ARTHROPLASTY
}

\author{
A. AVGERI ${ }^{1}$, S. SANDERS ${ }^{2}$, B. CINQUIN ${ }^{4}$, L. SEDEL ${ }^{1}$, P. BIZOT ${ }^{3}$ AND E. BUDYN $^{2}$ \\ ${ }^{1}$ CNRS-Inserm Laboratory of osteoarticular Biology, Bioimaging and Bioengineering B3OA \\ UMR7052 U1172, University of Paris \\ 75010, Paris, France \\ angelina.avgeri@gmail.com \\ ${ }^{2}$ Department of Mechanical Engineering - CNRS Laboratory of Mechanics and Technology LMT \\ UMR8535, Ecole Normale Supérieure Paris-Saclay, University Paris-Saclay \\ 91190 Gif-sur Yvette, France \\ ebudyn@ens-paris-saclay.fr
}

${ }^{3}$ Department of Othopedic surgery - CNRS-Inserm Laboratory of osteoarticular Biology, Bioimaging and Bioengineering B3OA UMR7052 U1172, University of Paris

75010 Paris, France

${ }^{4}$ CNRS Laboratory of Biology and Applied Pharmacology LBPA UMR8113, Ecole Normale Supérieure Paris-Saclay, University Paris-Saclay

91190 Gif-sur Yvette, France

Key words: Soft tissue modelling, Tissue mechanics, Hip capsule ligament, Total Hip Arthroplasty

\begin{abstract}
Total hip arthroplasty is a surgical procedure that replaces the hip joint by artificial materials. Here, the morphological and mechanical properties of the scar tissues that form around implants composed of either polymer and metal or ceramic are compared to native tissue removed during an initial total hip arthroplasty. Immuno-histological analyses of the samples showed different hierarchical structures of the tissues over three scales: the fiber, the fascicle and the tissue scales. At the tissue scale, micro-tensile tests were performed on millimetric samples and their non-linear elastic responses were identified by either an exponential law or an Ogden third-order constitutive model. At the fiber scale, a patient-specific micro-scale finite element model including the measured morphological parameters and the identified Ogden constitutive models for the fiber and for the matrix composed of a mixture of fibers in ground substance.
\end{abstract}

\section{INTRODUCTION}

Hip replacement is currently one of the most common orthopaedic surgeries. In a total hip arthroplasty surgery, the hip capsule ligament, a fibrous tissue that surrounds and stabilizes the hip joint is partially or completely removed, in order to have access to the hip joint [1]. Six to twelve weeks following a total hip arthroplasty, a fibrous tissue that is not identical to the native hip capsule ligament reforms. After a total hip arthroplasty, morphological and mechanical differences between native and neoformed 
capsule ligaments can be more or less pronounced depending on the material of the implant that has replaced the defective joint $[2,3]$. Nonetheless, the scar tissue that forms around the operated hip joint plays a role for the joint stability in particular in delaying the need for revision surgery [4]. During an total hip arthroplasty, the ligament can be either damaged or partially removed. It is therefore crucial to understand the mechanical support that the new tissue that forms after a total hip arthroplasty provides to the joint, in order to assess its stability. Previous studies on hip capsule specimen harvested in human cadavers have investigated the material properties of the iliofemoral and the ischio- femoral ligaments which are the most important capsule ligaments [5]. Native iliofemoral ligament restricts the extension of the hip while the ischiofemoral ligament restricts internal rotation. In the present study, native hip capsule ligament tissues were harvested in the iliofemoral ligament region during total hip arthroplasties.

To determine the constitutive behavior of hip capsule ligaments, animal studies have been performed that made it possible to calculate Poisson's ratios by direct measurements of the cross-sectional areas of the specimens [6]. Such methods based on cross-sectional area measurements, showed methods that the assumption of incompressibility lead to smaller errors in the calculation of true stress than in models that would assume a constant cross-section. Other models allow compressibility of the tissue. Such models were based on a strain energy density function that could describe the volume loss in ligaments. Large Poisson ratios that were experimentally measured were predicted by these models, as well as a stressstrain response of ligament tissues similar to the response of an incompressible model [7]. A model for compressible tissues lead to a linear stress-strain relationship when a shear stress was applied along the direction of the fibers. An exponential stress-strain relationship was suggested to be more appropriate.

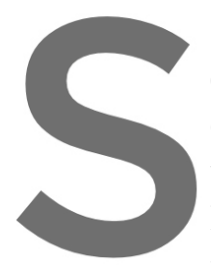

Soft fibrous tissues havd

energy density functions other models, soft tissues

terials $[9,10]$ using strai

laws or 3rd order statistic

ing the strain energy density
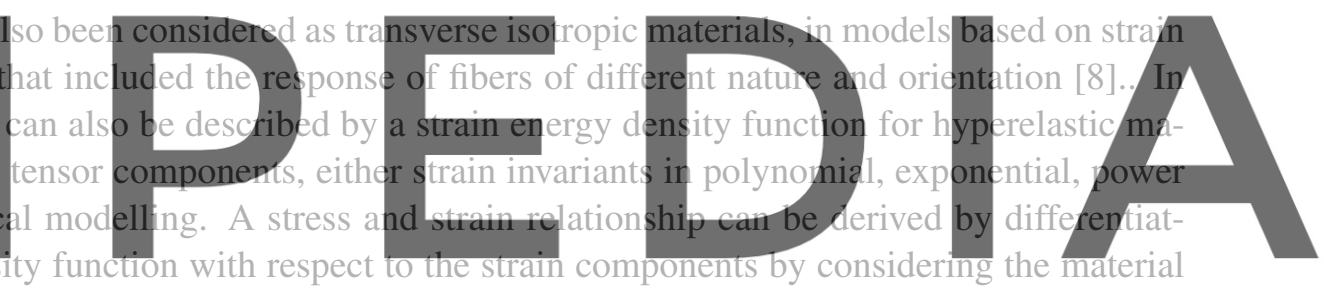

either as linear elastic in the case of small displacements [11] or nonlinear in the case of large defor-

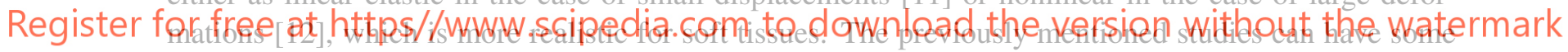

limitations pertaining either to the types of tissues, animal [5] or human cadavers versus fresh human

tissues, or to the assumptions in the continuum models, isotropic or anisotropic and isochoric $[8,13,12]$ or incompressible [7] versus anisotropic and compressible.

For fresh human hip capsule ligament tissues, we propose to first model our tissue as a uniform homogenized tissue described by an exponential strain energy density function and then identify the corresponding third order Ogden model [14]. To understand the behavior at the micro-scale, a fiber scale model with explicit patient-specific morphology of the fibers inside the ground substance is created from perturbation of the identified homogenized third order Ogden model. We first describe the experimental setting and the construction of the theoretical models. The results are then presented and analyzed in a following discussion. 


\section{MATERIALS AND METHODS}

\subsection{Tissue harvesting and morphological characterization}

Hip capsule ligament tissues were harvested during total hip arthroplasties and their orientation labelled. The tissues were then preserved at $4^{\circ} C$ in physiological serum and antibiotics $2 \mathrm{x}$. Tissue having formed around implants made of different materials were classified into 3 categories as follows: tissues formed around ceramic on ceramic implants were denoted "ceramic", tissues formed around implants containing polymer were denoted "polymer" and native tissues present prior to any total hip arthroplasty were denoted "native". For this study, the following implants were considered: a) Ceramic on ceramic (CoC): The stem and the socket were uncemented, the head and the insert of the socket were made of pure alumina (A12O3). These implants are usually inserted in active and/or young patients [15]; b) Metal on polyethylene (Met/PE): The stem and the socket were cemented (with PMMA). The head was made of stainless steel (not Cr-Co) and the socket was made of full UHMWPE (ultrahigh molecular weight PE) (conventional PE). These implants are of high durability and performance.

Two samples of neo-formed tissue grown around a Ceramic on Ceramic implant, three samples grown around implants containing polymer and three samples of tissue grown prior to any total hip arthroplasty were tested under tension. The samples originated from both male and female donors of the following age groups: 35-39 for patients with CoC implants, 55-80 for patients with Met/PE implants and 67-85 from patients without any implant.

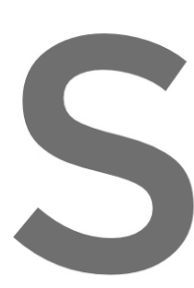

Immuno-histochemistry

in slices of $5 \mu \mathrm{m}$ thickness

were used: a) Hematoxy

Fluorescent Microscopy ot

served using bright-field

microscopy (Leica TCC SP8 Cor
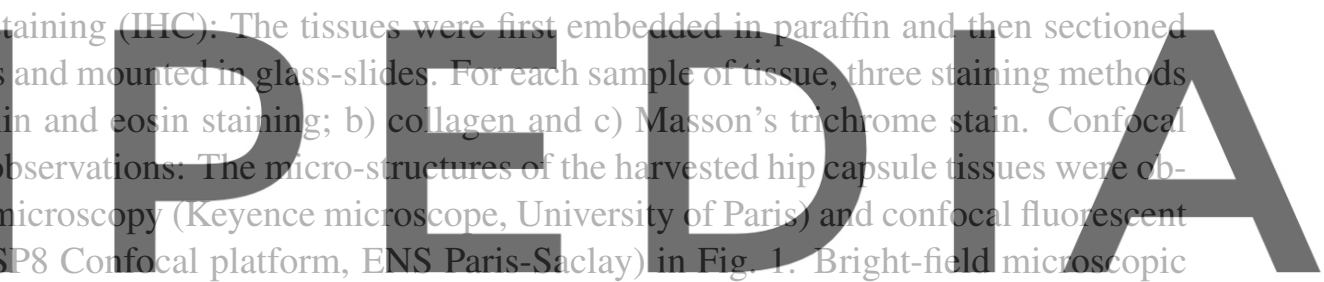

observations of immuno-histological slides revealed different hierarchical structures over three scales:

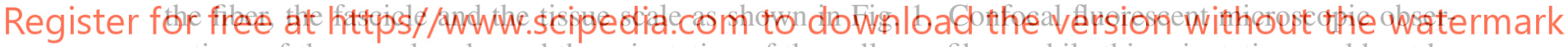
vations of the samples showed the orientation of the collagen fibers while this orientation could not be clearly detected using transmission bright-field optical microscopy. The collagen fibers were found to be oriented along the direction of the tissue fascicle.

\subsection{Experimental setting of the micro-tensile tests}

Micro-mechanical tensile tests were performed under increasing load with periodic load/unloading cycles. Millimeter size samples of the three types of hip capsule tissues were tested. The experimental set up is shown in Fig. 1. At each force increment, changes in the width and length of the tissue were measured. Only the initial thickness of the tissue could be measured. In the mathematical model, the tissue was assumed to be incompressible and its volume was assumed constant to calculate the thickness value that corresponds to each change in force.

For the homogenized macroscopic model, the hip capsule tissue was considered as a continuum, homogeneous non-linear elastic. Hip capsule ligament is known to be a multi-scale fibrous tissue with a preferential direction aligned with the fascicles, the tissue was represented by orthonormal bases, denoted 

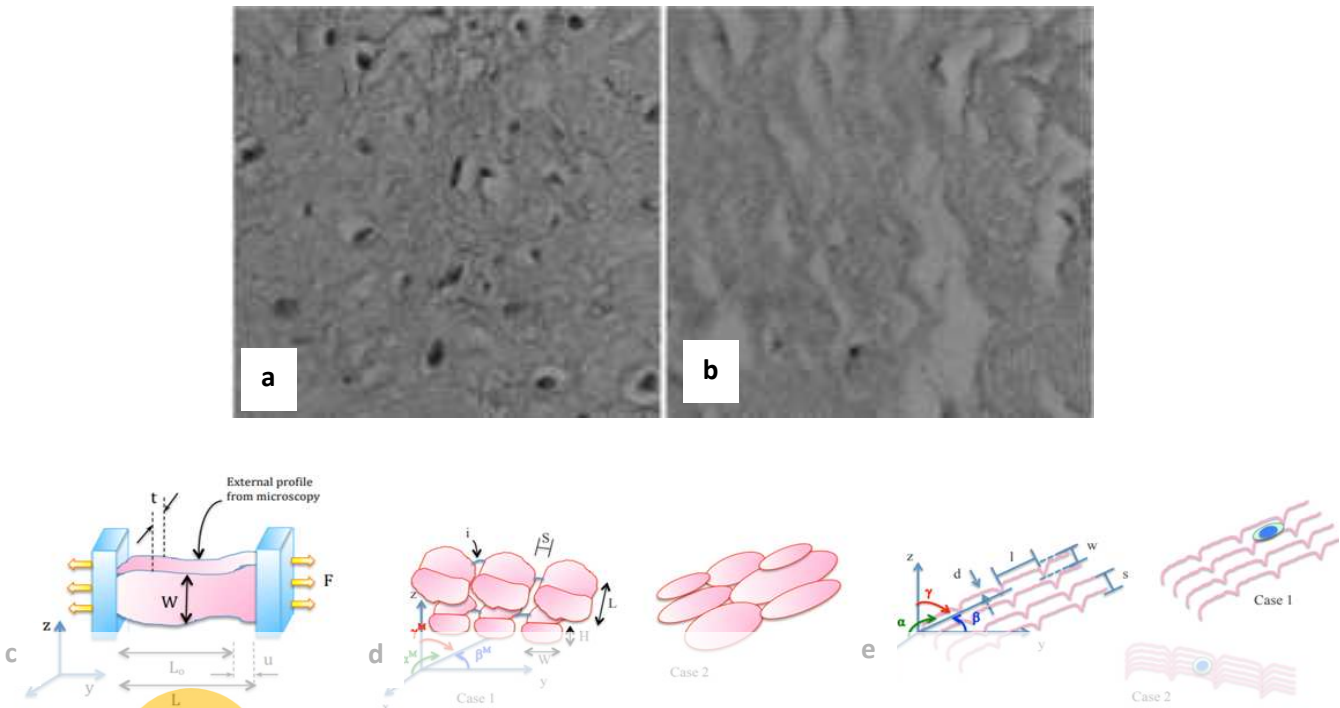

Figure 1: 2D Confocal fluorescent Microscopic observations of hip capsule scar tissues a) in the transverse plane and $\mathrm{b}$ ) in the longitudinal plane of sample 2 (ceramic) excited by a wavelength of $433 \mathrm{~nm}$ to collect the autofluorescence of Type I collagen, the image width is $80 \mu \mathrm{m}$; Schematic representation of the tissue: c) Macro-scale (tissue scale), d) Meso-scale (fascicle scale), e) Micro-scale (fiber scale);
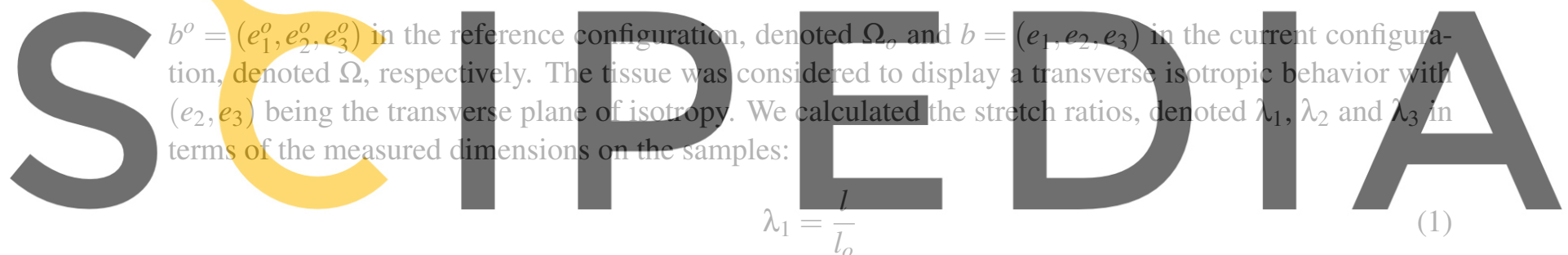

$$
\lambda_{1}=\frac{l}{l_{0}}
$$

Register for free at https//www.scipedia.com to download the version without the watermark

$$
\lambda_{3}=\frac{t}{t_{o}}
$$

The deformation gradient denoted $F$ calculated using a polar decomposition $F=R \cdot U$, with $R$ is the rotation matrix and $U$ is the right stretch tensor.

\subsection{Identification of the strain energy density function for an exponential description of the tissue macroscopic response}

The Strain Energy Density function was first assumed to follow an exponential description of which the parameters were directly extracted from the smoothed experimental force displacement curve shown in Fig. 3.

The second Piola-Kirchhoff was calculated as the conjugate of the Green-Langrange strain as follows

$$
\left.S(E)=\alpha_{1} e^{\left(-\lambda_{1} E_{11}\right.}\right)+\alpha_{2} e^{\left(-\lambda_{2} E_{11}\right)}-\left(\alpha_{1}+\alpha_{2}\right)
$$


with $E$ the Green-Lagrange strain. The Strain Energy Density function $W_{u}\left(E_{A}\right)$ was given by:

$$
W_{u}(E)=-\left(\alpha_{1}+\alpha_{2}\right) E-\left(\frac{\alpha_{1}}{\lambda_{1}}\left(e^{-\lambda_{1} E}-1\right)+\frac{\alpha_{2}}{\lambda_{2}}\left(e^{-\lambda_{2} E}-1\right)\right.
$$

In a preliminary approximation, the experimental setting was assumed to prevent any rotation $(R=I)$ as the samples were securely clamped at each extremity and the clamps were guided by perfectly parallel rails and the collagen fibers were assumed to be aligned with the tensile direction and this assumption was verified by confocal fluorescent microscopic observations of the samples prior to the micro-tension test. I leading to:

$$
F=\left[\begin{array}{ccc}
\lambda_{1} & 0 & 0 \\
0 & \lambda_{2} & 0 \\
0 & 0 & \lambda_{2}
\end{array}\right]
$$

in the case of transverse isotropy and

$$
t=\frac{t_{0} w}{w_{0}}
$$

where $c$ is the right Cauchy-Green deformation tensor and $U$ is the right stretch tensor. In our case:

$$
E=\frac{1}{2}\left[\begin{array}{ccc}
\lambda_{1}^{2}-1 & 0 & 0 \\
0 & \lambda_{2}^{2}-1 & 0 \\
0 & 0 & \lambda_{2}^{2}-1
\end{array}\right]
$$

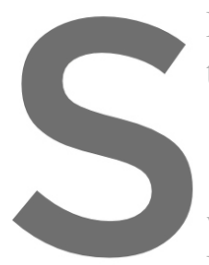

For stress measures, becau

tensor $U$ in our case, the firs

where $d f$ represents an infinitesim
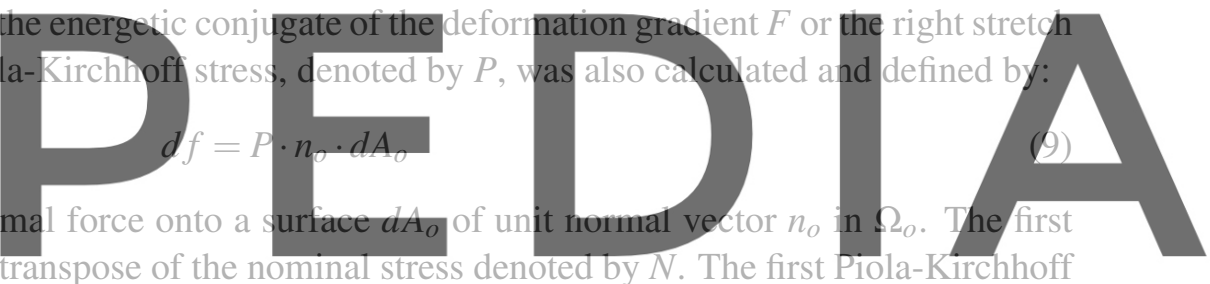

stress can be related to the Cauchy stress $\sigma$ that was also calculated as follows:

Register for free at https//www.scipedia.com to download the version without the watermark

$$
P=J \sigma \cdot F^{-T}
$$

where in our case of uni-axial tensile loading lead to:

$$
\sigma=\left[\begin{array}{ccc}
\frac{F_{1}}{w t} & 0 & 0 \\
0 & 0 & 0 \\
0 & 0 & 0
\end{array}\right]
$$

and

$$
P=\lambda_{1} \lambda_{2}^{2}\left[\begin{array}{ccc}
\frac{F_{1}}{w t} & 0 & 0 \\
0 & 0 & 0 \\
0 & 0 & 0
\end{array}\right]\left[\begin{array}{ccc}
\frac{1}{\lambda_{1}} & 0 & 0 \\
0 & \frac{1}{\lambda_{2}} & 0 \\
0 & 0 & \frac{1}{\lambda_{2}}
\end{array}\right]
$$

Using the pull-back of the infinitesimal force in the current configuration to the original configuration, the Second Piola-Kirchhoff stress, denoted $S$, was also calculated as the conjugated stress to the GreenLagrange deformation tensor $E$ as follows:

$$
S=J F^{-1} \cdot \sigma \cdot F^{-T}
$$


leading to:

$$
S=\lambda_{1} \lambda_{2}^{2}\left[\begin{array}{ccc}
\frac{1}{\lambda_{1}} & 0 & 0 \\
0 & \frac{1}{\lambda_{2}} & 0 \\
0 & 0 & \frac{1}{\lambda_{2}}
\end{array}\right]\left[\begin{array}{ccc}
\frac{F_{1}}{w t} & 0 & 0 \\
0 & 0 & 0 \\
0 & 0 & 0
\end{array}\right]\left[\begin{array}{ccc}
\frac{1}{\lambda_{1}} & 0 & 0 \\
0 & \frac{1}{\lambda_{2}} & 0 \\
0 & 0 & \frac{1}{\lambda_{2}}
\end{array}\right]
$$
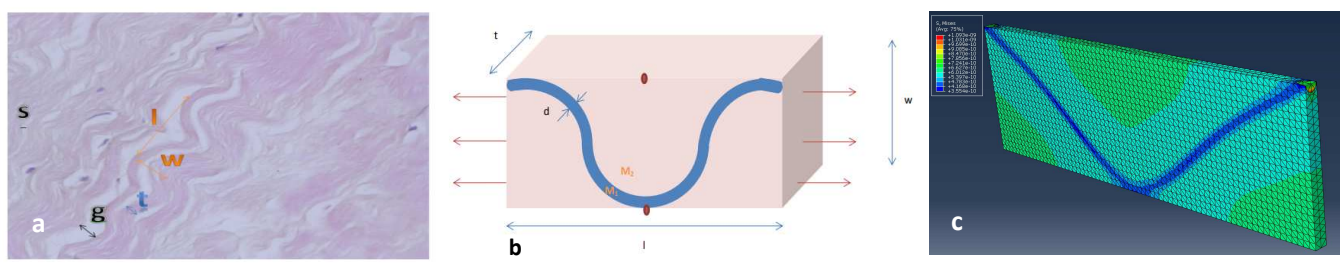

Figure 2: Construction of single fiber unit-cell model: a) Characteristic parameters of the fiber wave motif, b) Schematic representation of the collagen fiber, c) FEM patient-specific single fiber unit cell model.

From the calculation of the previously described stress and strain measures calculated at each point of our experimental data sets, an exponential constitutive law was derived for each tensile test performed on each type of tissue sample [16]. The curves are shown in Fig. 3 and follow the form:
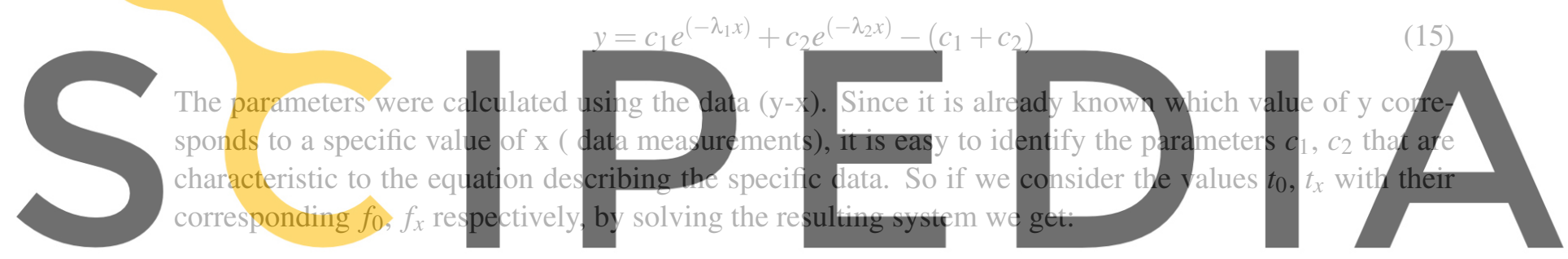

Register for free at https//www.scipedia.compto downloath the version without the watermark

and

$$
c_{1}=\frac{f_{0}-c_{2} e^{-\lambda_{2} t_{0}}}{e^{-\lambda_{1} t_{0}}}
$$

Differences among the different tissue types appeared. The characteristic parameters for each tissue type were examined separately and a representative curve of each tissue was later isolated.

Note that $L_{\min }$ of the tissue during the mechanical tests was specified as the one corresponding to the minimum force, $F_{\min }$. The corresponding minimum width, $W_{\min }$, was also identified and the tensile displacement of the tissue was calculated as follows:

$$
U_{1}=L-L_{\min }
$$

The minimum force was determined by its minimum absolute value, so that it would correspond to the length measurement closest to the resting length value of the tissue corresponding to the value of $F$ closest to zero. Two adjustments could also be performed:

- For points corresponding to a length smaller than Lmin the displacements were shifted. 
- If the minimum displacement value was less than -0.2 , points were eliminated.

The parameters of the exponential law were identified for each test.

\subsection{Microscopic model based on the patient-specific fiber morphology}

Patient-specific and implant-specific morphological differences were quantified by characteristic patientspecific biological parameters such as the amplitude and the period of the fiber wave motif. The dimensions were defined as shown in Fig. 2 a). The average of their values for the different implant materials are given in Table 1. Differences between parameters provide insights in the way the fibers have formed in each tissue. The biological parameters were imported into the numerical model of a single collagen

Table 1: Biological parameters for loose and dense tissue samples: 1 denoted the fiber wave period, w the fiber wave amplitude, $\mathrm{g}$ is the distance between multiple fiber groups and $t_{g}$ denotes a fiber group thickness. Parameters could be different for loose or dense tissues. Therefore $s, g$, $t_{g}$ did not exist for Met/PE.

\begin{tabular}{ccccccc} 
Implant Material & $1(\mu \mathrm{m})$ & $\mathrm{w}(\mu \mathrm{m})$ & $\mathrm{d}(\mu \mathrm{m})$ & $\mathrm{s}(\mu \mathrm{m})$ & $\mathrm{g}(\mu \mathrm{m})$ & $\operatorname{tg}(\mu \mathrm{m})$ \\
\hline Native & 45.68 & 17.35 & 0.87 & 4.41 & 12.19 & 15.58 \\
\hline CoC & 24.97 & 7.53 & 0.69 & 6.32 & 18.80 & 9.09 \\
\hline Met/PE & 23.87 & 12.38 & 0.56 & - & - & -
\end{tabular}

fiber immersed in the mixture of other collagen fibers and ground substance as in [17] using Abaqus [18].

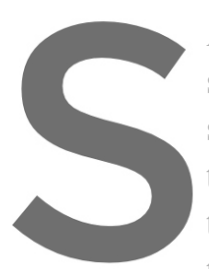

A representative patien

sured in the microscopic

shown in the Fig. 2 b) a

tified for the homogeniz

the tissue was transvers

third order Odgen material is as follows:
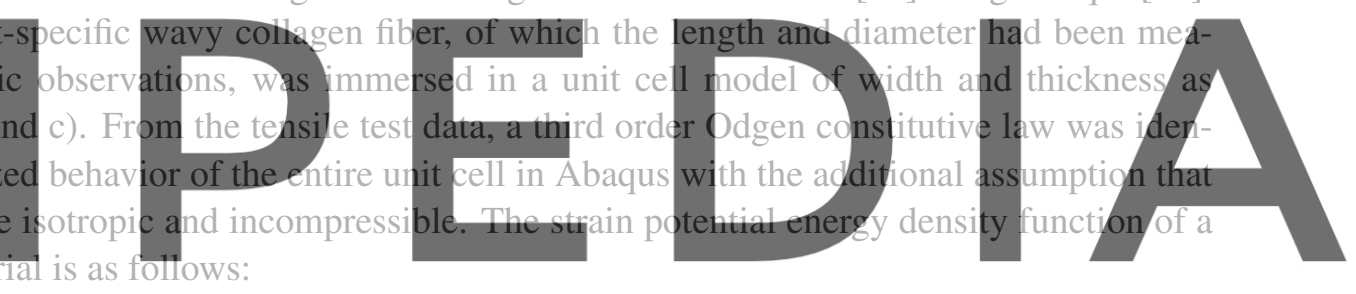

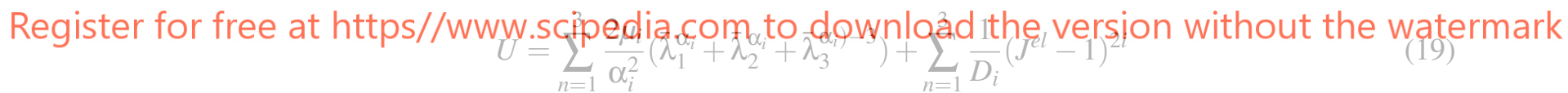

$$
\begin{gathered}
D_{i}=0 \\
\bar{\lambda}_{i}=J^{-\frac{1}{3}} \lambda_{i} \\
\lambda_{2}=\lambda_{3}=\lambda_{1}^{-\frac{1}{2}} \\
\lambda_{1}=1+\varepsilon \\
N=P^{T}=\sum_{1}^{3} \frac{2 \mu_{i}}{\alpha_{i}}\left(\lambda_{1}^{\alpha_{i}-1}-\lambda_{1}^{-\frac{\alpha_{i}}{2}-1}\right)
\end{gathered}
$$

where $\lambda_{i}$ are the principal stretches, $\alpha_{i}, \mu i$ and $D_{i}$ are material parameters. In equation $23, \varepsilon=\lambda-\mathbf{1}$ is the nominal strain, and $N$ is the nominal stress [14].

After identification of the parameters of the Ogden model for the homogenized equivalent material, the constitutive laws of the two-phase material in the unit cell were identified. The constitutive Ogden models 
Table 2: Ogden coefficients of the single fiber model for one material.

\begin{tabular}{ccccccc} 
Implant Material & $\alpha_{1}$ & $\alpha_{2}$ & $\alpha_{3}$ & $\mu_{1}$ & $\mu_{2}$ & $\mu_{3}$ \\
\hline Native & 23.92 & 25.00 & 21.81 & $-3.75 \mathrm{E}-02$ & $2.49 \mathrm{E}-02$ & $1,26 \mathrm{E}-02$ \\
\hline CoC & 2.00 & 4.00 & -2.00 & $-3.18 \mathrm{E}-02$ & $2.16 \mathrm{E}-02$ & $1.02 \mathrm{E}-02$ \\
\hline Met/PE & 23.75 & 25.00 & 21.30 & $-6.71 \mathrm{E}-02$ & $4.47 \mathrm{E}-02$ & $2,24 \mathrm{E}-02$
\end{tabular}

of the fiber and the matrix composed of a mixture of collagen and ground substance were calibrated in order to return the same mechanical response as the homogenized model. For each type of tissue (native, ceramic, polyethylene), the fiber volume fraction $f_{f}$ and the matrix volume fraction $f_{m}$ were calculated and used in a mixing rule to calculate the phase parameter $\mu$ as follows:

$$
\begin{gathered}
f_{f}=\frac{V_{f}}{V_{t o t}} \\
f_{m}=\frac{V_{m}}{V_{t o t}} \\
\mu=f_{f} 2 \mu+f_{m} x \mu
\end{gathered}
$$

The effect of the microscopic morphology was included by perturbing the identified Ogden coefficients. The material parameters $\alpha_{i}$ were left unchanged for both the fiber and the matrix. The Ogden coefficients are given in the Tables 3 and 2.
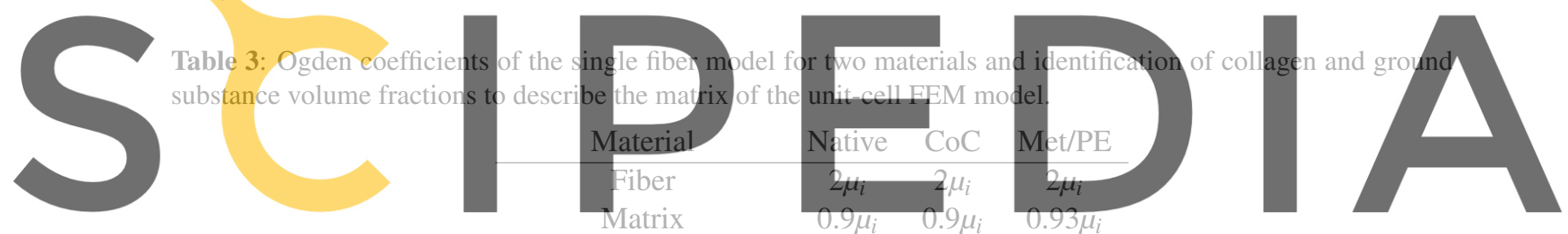

Volume fraction Native CoC Met/PE

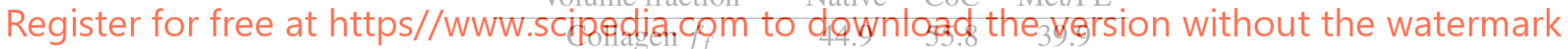

$$
\text { Ground substance } f_{m} \quad 55.1 \quad 44.2 \quad 60.1
$$

The volume fractions of ground substance and collagen were also calculated in the histology bright-field microscopic observations for each tissue type shown in Table 3.

The stresses of the unit cell single-fiber model were calculated from the separate stresses in the fiber and in the matrix using a mixing rule as shown in 28 :

$$
N=P^{T}=f_{f} N^{f}+f_{m} N^{m}
$$

Where $N^{m}$ is the nominal stress for the fiber and $N^{m}$ the nominal stress for the matrix. 


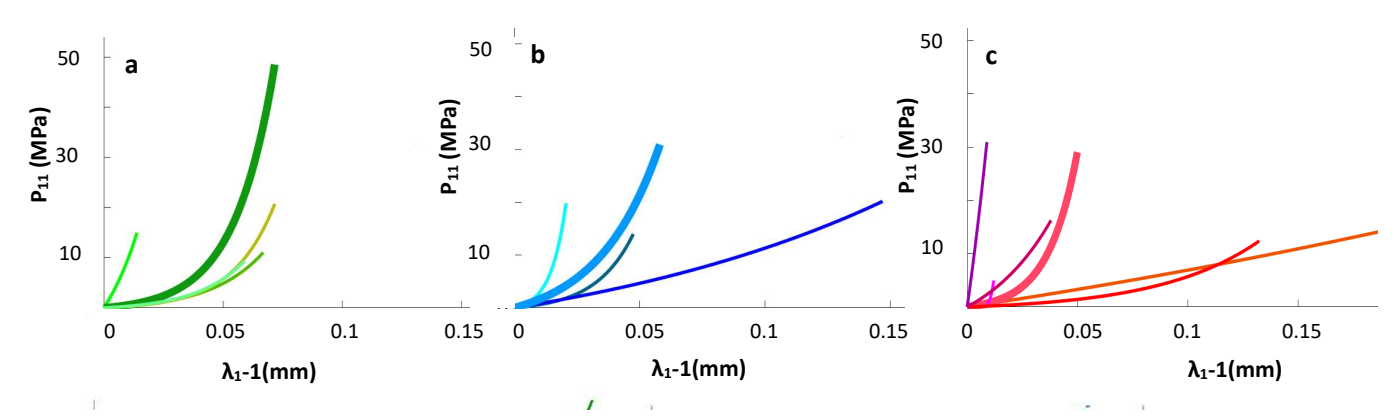

Figure 3: First Piola-Kirchhoff versus nominal strain tensile response for three different tissue types: a) Native tissues, b) tissues around $\mathrm{CoC}$ implants, c) tissues around Met/PE implants.

\section{RESUITS AND DISCUSSION}

\subsection{Macro scale exponential constitutive model identification for human hip capsule ligament scar tissues}

The morphological and mechanical properties of the hip capsule tissue, that forms near hip implants made of different materials after a total hip arthroplasty were compared to native tissue. Surgeons had visually observed differences between these tissue types and these morphological and mechanical disparities were

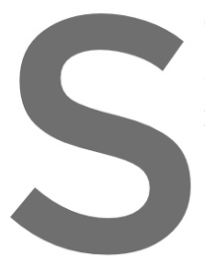
confirmed in the presents internal rotations [19] responses of the tissues staining of the samples scales

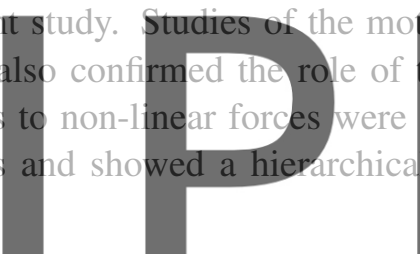

Hip capsule ligaments we
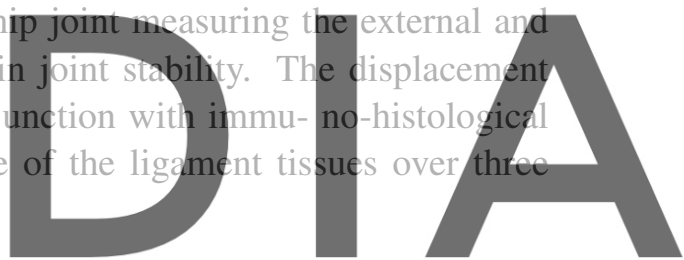
aments were treated as tensile bands to evaluate their contribution to the human knee.joint stiffness [201.

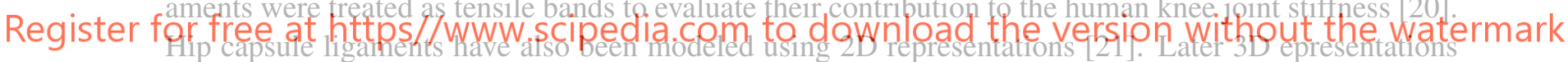
were developed to investigate the human ligament response to shear stress and was found to be highly visco-elastic [22]. Due to the complexity of the material, a 3D representation of the complete hip capsule had been suggested to be highly relevant to characterize and predict the role of the capsule on the joint using a finite element model [23]. Therefore, we propose a multi-scale 3D model of the hip capsule tissue including the patient-specific fiber morphology. The present study includes the tissue macroscopic response and the unit cell fiber model as a preliminary study that will be further complemented by a meso-scale model to study the interaction between fibers. The current preliminary model will make it possible to gain insight on the effect of the fiber morphology on the overall mechanical behavior of the hip capsule tissue.

\subsection{Micro scale Odgen constitutive model of human hip capsule ligament scar tissues}

A patient-specific finite element model was constructed for a single collagen fiber immersed in a matrix composed of collagen and ground substance. The hip capsule tissue displayed a third-order Ogden nonlinear elastic constitutive law often characteristic of the mechanical behaviour of biological tissues [24, 
25]. Three typical types of loading regimes were determined as shown in Fig. 4 a), b) and c). This unit cell model can show the effect of the progressive stretch of a wavy fiber on the overall unit cell deformation. The three regimes are typical of fibrous soft tissue non-linear elastic behavior and are decomposed into: 1- an initial loading regime in which the fibers are still relaxed, 2- a initial stretching regime, c- a fully stretched regime shown in Fig. 4 a), b) and c). At the mid-point of each region, the corresponding strain through a Dirichlet boundary condition was applied to the FEM unit cell model and the conjugated stress was calculated by volume average of the stress field in the FEM model. The mid-point of each region were determined by the intersection of the tangents at each extremity of the region.

The Von Mises stress fields at the mid-point of the three loading regimes are shown in Fig. 4 a), b) and c). The highest level of stress is exhibited in the native tissues. Medium stress level is displayed in the tissue formed near Met/PE implants and the lowest stress level is shown in tissue grown near CoC implants. The stiffness of each non-linear material is the slope in the stress-strain curve. The collagen fibers are stiffer than the matrix phase but exhibit lower stresses than the matrix because in the proposed model only half of the cross-section of the fiber is subjected to a prescribed displacement. These stress fields provide an insight to the mechanical behavior of the constituents of the hip capsule ligament at the microscale for which more studies are still needed, while the macroscale behavior has been more studied [26]. A proof of concept has been presented in this preliminary study and will be further completed by studies on groups of closer age of both genders.

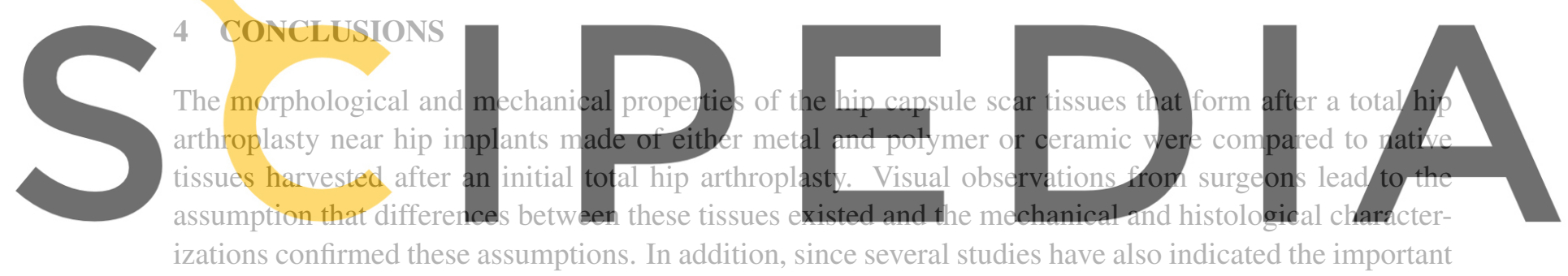

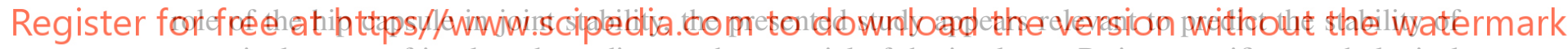

a particular type of implant depending on the material of the implants. Patient-specific morphological parameters measured in histological slides were implemented into a FEM micro-scale model to determine their effect on the overall mechanical behavior of the tissue. A series of uni-axial tensile micromechanical tests showed the non-linear elastic behavior of the fibrous tissues that were first identified by an exponential and then a third-order Ogden homogenized continuum mechanics model at the tissue scale level. Applying perturbations to the third-order Ogden macroscopic homogenized model lead to the determination of two third-order Odgen models for the collagen fiber and the matrix in which the fibers were embedded at the micro scale. The microscopic model exhibited the effect of the microscopic morphology wavy fiber on the overall tissue behavior calculated at the mid-point of three characteristic fiber loading regimes. These results could contribute to guide the choice by surgeons of patient-specific materials for a hip implant according to biomarkers such as age, sex, weight or physical activity of the patient. Because hip joint dislocation is one of the most common reasons for a revision surgery after a first total hip arthroplasty, our study has shown that the mechanical properties of the hip capsule scar tissue could play a decisive role in maintaining the joint stability. 

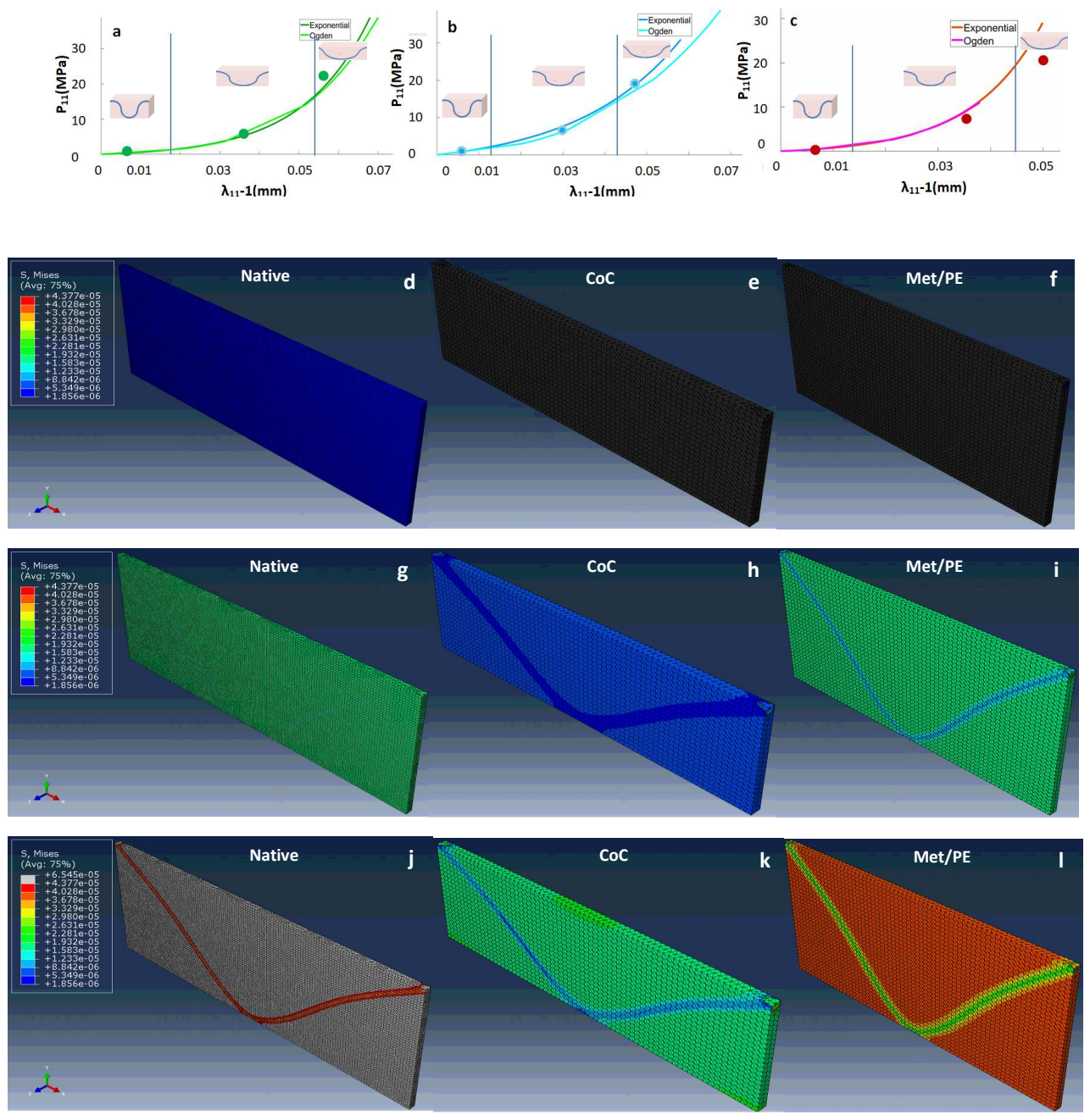

Figure 4: Exponential macroscopic model (solid lines) and homogenized Ogden model (darker solid lines) and FEM microscopic model response at the mid-locations of the three different fiber loading regimes at the dot points for a) the native tissue, b) the tissues formed around $\mathrm{CoC}$ implant and c) around Met/PE implants. The three points represent the stress and strain of FEM two-phase materials described by two different third order Ogden models. d)-l) Von Mises stress fields in the three types of tissues (Native, scar tissues formed near either ceramic or Metal/Polymer implants) subjected to the displacements at the mid-points of the three types of loading regimes.

\section{REFERENCES}

[1] R.J. van Arkel, K.C. Ng, S.K. Muirhead-Allwood, and R.T.J. Jeffers. Capsular ligament function after total hip arthroplasty. The Journal of bone and joint surgery, 100(14):1-10, 2018.

[2] J. Parvizi, L. Sedel, and M.J. Dunbar. Clinical faceoff: Instability after total hip arthroplasty: The potential role of the bearing surface. Clin Orthop Relat Res, page 476(4):678683, 2018.

[3] D. Hannouche, F. Devriez, J. Delambre, F. Zadegan, I. Tourabaly, L. Sedel, S. Chevret, and R. Nizard. Ceramic-onceramic tha implants in patients younger than 20 years. J Bone Joint Surg Am, page 474:520527, 2016. 
[4] K.J.Bozic, S.M Kurtz, E. Lau, K. Ong, T.P. Vail, and D.J.Berry. The epidemiology of revision total hip arthroplasty in the united states. J Bone Joint Surg Am, pages 91(1):128133,, 2009.

[5] J. D. Hewitt, R. R. Glisson, F. Guilak, and T. Parker Vail. The mechanical properties of the human hip capsule ligaments. The Journal of Arthroplasty, 17, 2001.

[6] C. Vergari, P. Pourcelot, L. Holden, G. Gerard B. Ravary-Plumioen, P. Laugier, D. Mitton, and N. Crevier-Denoix. True stress and poisson's ratio of tendons during loading. Journal of Biomechanics, pages 719-724, 2011.

[7] A.M. Swedberg, S.P. Reese, B.J. Ellis S.A. Maas, and J.A. Weiss. Continuum description of the poisson's ratio of ligament and tendon under finite deformation. Journal of Biomechanics, pages 3201-3209, 2014.

[8] G.A. Holzapfel. Biomechanics of Soft Tissue. Academic Press, Handbook of Material Behavior, 2000.

[9] N.Famaey and J.V. Sloten. Soft tissue modelling for applications in virtual surgery and surgical robotics. Computer Methods in Biomechanics and Biomedical Engineering, pages 11:4, 351-366, 2008.

[10] R.C. Hibbeler. Mechanics of materials. Pearson Prentice Hall, 2011.

[11] R.L Armentano, J.G. Barra, J. Levenson, A. Simon, and R.H. Pichel. Arterial wall mechanics in conscious dogs. assessment of viscous, inertial, and elastic moduli to characterize aortic wall behavior. Circ. Res., page 76(3):468478, 1995.

[12] G.A. Holzapfel, R. Eberlein, P. Wriggers, and H.W.Weizscker. Large strain analysis of soft biological membranes: Formulation and finite element analysis. Computer Methods in Applied Mechanics and Engineering, pages 11:4, 351366, 1996.

[13] D. Garcia-Gonzalez, A. Jrusalem, S. Garzon-Hernandez, R. Zaera, and A. Arias. A continuum mechanics constitutive framework for transverse isotropic soft tissues. Journal of the Mechanics and Physics of Solids, 112, 2017.

[14] R.W. Odgen. Clarge deformation isotropic elasticity on the correlation of theory and experiment for incompressible rubber-like solids. Proceedings of the Royal Society of London. Series A, Mathematical and Physical Sciences, 326(1567):565584, 1972.

[15] M.Merola and S. Affatato. Materials for hip prostheses: A review of wear and loading considerations. Materials(Basel), 12(3):495, 2019.

[16] H.Choi and R. Vito. Two-dimensional stressstrain relationship for canine pericardiu. J Biomechl Eng, page 112(2):153159, 1990.

[17] S. Bhogle, G. Mistri, B. Vesper S. Lacey, J. Radosevich, M. Colvard, and E. Budyn. Identification of cell microenvironment in porcine skine laser cauterization. CMAME, 314:19-45, 2017.

[18] M.Smith. ABAQUS/Theory Manual, Version 6.6, Large strain elasticity. Dassault Systèmes Simulia Corp., United States, 2009.

[19] H.D.Martin, A. Savage, B.A. Braly, I.J. Palmer, D.P. Beall, and B.T. Kelly. The function of the hip capsular ligaments: a quantitative report. arthroscopy. Epub, 24(2):188-95, 2008.

[20] M.S. Hefzy and E.S. Grood. An analytical technique for modeling knee joint stiffnesspart ii: Ligamentous geometric nonlinearities. J Biomech Eng., 105(2):145-153, 1983.

[21] K.W. Simbeya, N. Shrive, C.B Frank, and J.R. Matyas. A micromechanical finite element model of rabbit medial collateral ligament. recent advances in computer methods in biomechanics and biomedical engineering. page 240249, 1992.

[22] J.A.Weiss, J.C. Gardiner, and C. Bonifasi-Lista. Ligament material behavior is nonlinear, viscoelastic and rateindependent under shear loading. J Biomech, page 943950, 2002.

[23] K.J. Stewart, D.R. Pedersen, J.J. Callaghan, and T.D. Brown. Implementing capsule representation in a total hip dislocation finite element model. Orthopaedic Journal, 24: 18, 2004.

[24] C. Wex, S. Arndt, A. Stoll, C. Bruns, and Y. Kupriyanova. Isotropic incompressible hyperelastic models for modelling the mechanical behaviour of biological tissues: a review. Biomedical Engineering / Biomedizinische Technik, 60:6, 2015.

[25] K. Laksari, M. Shafieian, and K. Darvish. Constitutive model for brain tissue under finite compression. Journal of Biomechanics, 45:4:642-646, 2012.

[26] C.F.Guimares, L. Gasperini, A.P. Marques, and R.L. Reis. The stiffness of living tissues and its implications for tissue engineering. Nature Reviews Materials, page 351370, 2020. 